\title{
High Energy Gamma Rays from Nebulae Associated with Extragalactic Microquasars and Ultra-Luminous X-ray Sources
}

\author{
Yoshiyuki Inoue $^{\mathrm{a}}$, Shiu-Hang Lee ${ }^{\mathrm{a}, \mathrm{b}}$, Yasuyuki T. Tanaka ${ }^{\mathrm{c}}$, Shogo B. Kobayashi ${ }^{\mathrm{d}}$ \\ ${ }^{a}$ Institute of Space and Astronautical Science JAXA, 3-1-1 Yoshinodai, Chuo-ku, Sagamihara, Kanagawa 252-5210, Japan \\ ${ }^{b 2}$ Department of Astronomy, Kyoto University, Kitashirakawa, Sakyo-ku, Kyoto 606-8502, Japan \\ ${ }^{c 3}$ Hiroshima Astrophysical Science Center, Hiroshima University, 1-3-1 Kagamiyama, Higashi-Hiroshima, Hiroshima 739-8526, Japan \\ ${ }^{d}$ Department of Physics, The University of Tokyo, 7-3-1 Hongo, Bunkyo-ku, Tokyo 113-0033, Japan
}

\begin{abstract}
In the extragalactic sky, microquasars and ultra-luminous X-ray sources (ULXs) are known as energetic compact objects locating at off-nucleus positions in galaxies. Some of these objects are associated with expanding bubbles with a velocity of 80-250 $\mathrm{km} \mathrm{s}^{-1}$. We investigate the shock acceleration of particles in those expanding nebulae. The nebulae having fast expansion velocity $\gtrsim 120 \mathrm{~km} \mathrm{~s}^{-1}$ are able to accelerate cosmic rays up to $\sim 100 \mathrm{TeV}$. If $10 \%$ of the shock kinetic energy goes into particle acceleration, powerful nebulae such as the microquasar S26 in NGC 7793 would emit gamma rays up to several tens TeV with a photon index of $\sim 2$. These nebulae will be good targets for future Cherenkov Telescope Array observations given its sensitivity and angular resolution. They would also contribute to $\sim 7 \%$ of the unresolved cosmic gamma-ray background radiation at $\geq 0.1 \mathrm{GeV}$. In 'contrast, particle acceleration in slowly expanding nebulae $\lesssim 120 \mathrm{~km} \mathrm{~s}^{-1}$ would be less efficient due to ion-neutral collisions and result in softer spectra at $\gtrsim 10 \mathrm{GeV}$.
\end{abstract}

'Keywords: astroparticle physics, ISM: bubbles, gamma rays: ISM, stars: black holes

\section{Introduction}

In the very high energy (VHE; $\gtrsim 50 \mathrm{GeV}$ ) gamma-ray sky, a few hundreds of objects have been detected by the Large 'Area Telescope (LAT) on board the Fermi gamma-ray telescope '(Fermi) [7] and by the imaging atmospheric Cherenkov telescopes [see e.g. 84]. Further progress is anticipated in the near future by the Cherenkov Telescope Array (CTA) [8]. Its improved flux sensitivity and angular resolution will enable us to unveil new particle accelerators in the Universe.

In the extragalactic sky, various source classes have been considered for future CTA observations such as active galactic nuclei [76], gamma-ray bursts [38], star forming galaxies [3], and cluster of galaxies [3]. Here, the angular resolution of CTA will achieve $\lesssim 3 \operatorname{arcmin}$ at $\gtrsim 1 \mathrm{TeV}^{1}$. As angular sizes 'of nearby galaxies up to $\sim 10 \mathrm{Mpc}$ is $\sim 10 \mathrm{arcmin}$, it would be possible to spatially resolve particle accelerators in those galaxies.

It would be difficult to detect supernova remnants or pulsar wind nebulae in extragalactic galaxies considering their luminosities $\left(L_{\gamma} \lesssim 10^{36} \mathrm{erg} \mathrm{s}^{-1}\right.$ ). Here, some galaxies are known to host more powerful compact objects such as microquasars and ultra-luminous X-ray sources (ULX) whose kinetic or radiative power is $\gtrsim 10^{39} \mathrm{erg} \mathrm{s}^{-1}$. These objects are discovered at off-nucleus positions [27].

Microquasars are X-ray binary systems having relativistic bipolar outflows or jets whose kinetic power is greater than $10^{39} \mathrm{erg} \mathrm{s}^{-1}$ [63]. ULXs are also compact X-ray binary systems having X-ray luminosities greater than $10^{39} \mathrm{erg} \mathrm{s}^{-1}$ [27]. Although X-ray emission mechanisms from microquasars and ULXs are not fully understood yet ${ }^{2}$, some of them are known to be associated with expanding nebulae with a velocity of $80-250 \mathrm{~km} \mathrm{~s}^{-1}$ and a size of $\sim 200 \mathrm{pc}[20,69]$. Since the kinetic power of those nebulae is known to be comparable to or even greater than the radiative or jet kinetic power [20], the nebulae are good candidates as new cosmic-ray acceleration sites and possibly they would emit gamma rays through hadronuclear interactions with ambient gases.

In this paper, we investigate the diffusive shock acceleration of particles in the expanding nebulae associated with extragalactic microquasars and ULXs. And, we estimate expected gamma-ray and neutrino signals from those nebulae. We further consider their contribution to the cosmic gamma-ray and neutrino background radiation and future detectability by CTA. Throughout this paper, we define $Q_{x}=Q / 10^{x}$.

\footnotetext{
Email addresses: yinoue@astro.isas.jaxa.jp (Yoshiyuki Inoue), herman@kusastro.kyoto-u.ac.jp (Shiu-Hang Lee), ytanaka@hep01.hepl.hiroshima-u.ac.jp (Yasuyuki T. Tanaka),

kobayashi@juno.phys.s.u-tokyo.ac.jp (Shogo B. Kobayashi) mass of $M_{\mathrm{BH}} \gg 10 M_{\odot}$ [e.g. 59], supercritical mass acce
${ }^{1}$ https://portal.cta-observatory.org/Pages/CTA-Performance.aspblack holes [e.g. 30, 51], or accreting pulsars [e.g. 11].

${ }^{2}$ There are three distinct ideas widely considered to interpret high X-ray luminosities of ULXs, although there is no general agreement on their nature; sub- or trans-Eddington accretion onto intermediate mass black holes with a mass of $M_{\mathrm{BH}} \gg 10 M_{\odot}$ [e.g. 59], supercritical mass accretion onto stellar mass
} 


\section{X-ray Source Embedded Bubbles in the local Universe}

The size of the microquasar and ULX bubbles $R_{b}$ is of an order of $200 \mathrm{pc}$ and the expansion velocity of the bubbles is known to be $v_{s}=80-250 \mathrm{~km} \mathrm{~s}^{-1}[20,69]$. Following the selfsimilar expansion law $[47,86]$, the bubble size can be described as

$$
R_{b} \approx 0.76\left(P_{\mathrm{kin}} / \mu m_{p} n_{\mathrm{gas}}\right)^{1 / 5} t^{3 / 5} \sim 200 P_{\mathrm{kin}, 40.5}^{0.2} n_{\mathrm{gas}, 0.5}^{-0.2} t_{13.5}^{0.6} \mathrm{pc},
$$

where $P_{\text {kin }}$ is the time-averaged kinetic power, $\mu=0.61$ is the mean molecular weight, $m_{p}$ is the proton mass, $n_{\text {gas }}$ is the gas density, and $t$ is the age of the system. Thus, the characteristic age of the bubble is

$$
\tau=3 R_{b} / 5 v_{s} \sim 4.9 \times 10^{5} R_{b, 20.8} v_{s, 7.1}^{-1} \mathrm{yr} .
$$

This age is comparable to the expected ULX lifetime [e.g. $42,62]$. Once we measure the bubble size and the expanding velocity, the time-averaged kinetic power of the bubble can be described as

$$
P_{\text {kin }} \approx 18 \mu m_{p} n_{\text {gas }} R_{b}^{2} v_{s}^{3} \sim 3.6 \times 10^{40} R_{b, 20.8}^{2} v_{s, 7.1}^{3} n_{\text {gas }, 0.5} \mathrm{erg} \mathrm{s}^{-1} .
$$

The Mach number of the shock due to the expanding nebulae is estimated as

$$
\mathcal{M} \approx v_{s} /\left(\gamma k_{B} T_{u} / \mu m_{p}\right)^{1 / 2} \sim 8.0 v_{s, 7.1} T_{u, 4}^{-1 / 2},
$$

where $\gamma=5 / 3$ is the specific heat ratio, $k_{B}$ is the Boltzmann constant, and $T_{u}$ is the temperature of the upstream of the shock. $\mathcal{M}$ is larger than unity for the upstream cold gas component with $T_{u}<10^{4} \mathrm{~K}$. The dynamical timescale in which the shock dissipates is

$$
t_{\mathrm{dyn}} \approx \frac{R_{\mathrm{b}}}{v_{s}} \sim 8.1 \times 10^{5} R_{b, 20.8} v_{s, 7.1}^{-1} \mathrm{yr} .
$$

The downstream temperature is estimated as

$$
T_{d} \approx 2(\gamma-1) \mu m_{p} v_{s}^{2} / k_{B} /(\gamma+1)^{2} \sim 2.0 \times 10^{5} v_{s, 7.1}^{2} \mathrm{~K} .
$$

Thus, radiative cooling plays an important role. For a solar metallicity interstellar medium (ISM) environment, the cooling time scale is given by [Eq. 34.4 of 24]

$$
t_{\mathrm{rad}} \sim 1.4 \times 10^{3} n_{\text {gas }, 0.5}^{-1} v_{s, 7.1}^{17 / 5} \mathrm{yr},
$$

for $10^{5} \lesssim T \lesssim 10^{7.3} \mathrm{~K}$. If the metallicity is $0.1 Z_{\odot}$, the time scale will become a factor of 3 longer. From Equation 5 and Equation 7, the downstream plasma cools within dynamical timescale. In other words, these nebula shocks are radiative. UV photons from the radiative zone would significantly ionize the upstream ISM. At $v_{s} \gtrsim 120 \mathrm{~km} \mathrm{~s}^{-1}$, shock induced ionizing radiation is strong enough to completely ionize the upstream gas $[36,56,74]$. At the downstream, the temperature decreases and the density increases as the radiation cools the gas in the downstream. As the shock compression ratio $r=(\gamma+1) \mathcal{M}^{2} /\left[(\gamma-1) \mathcal{M}^{2}+2\right] \sim 4$ in our case, we assume the spectral index of 2 for simplicity in this paper.
Here, the magnetic field in the shock upstream can be described as

$$
B_{u}=1.7 b \sqrt{n_{\mathrm{gas}, 0.5}} \mu \mathrm{G},
$$

where the dimensionless parameter $b$ is $\sim 1$ from Zeeman measurements of self-gravitating molecular clouds in the Galaxy [19]. The magnetic field in the downstream region will be amplified by compression as

$$
B_{d}=\sqrt{\frac{1}{3}+\frac{2}{3} r^{2}} B_{u} \sim 5.7 n_{\mathrm{gas}, 0.5}^{1 / 2} \mu \mathrm{G} .
$$

Here, if Alfvén-wave turbulence is fully generated, $B_{d}$ can be amplified locally around the shocks [see e.g. 1214, 75]. In such cases, $B_{d}$ would become as high as $B_{d} \approx$ $\left(4 \pi \xi_{B} f_{\text {ion }} \mu n_{\text {gas }} m_{p} v_{s}^{2}\right)^{1 / 2} \sim 74 \xi_{B, 0}^{1 / 2} f_{\text {ion }, 0}^{1 / 2} n_{\text {gas }, 0.5}^{1 / 2} v_{s, 7.1} \mu \mathrm{G}$, where $\xi_{B}$ is the magnetic field amplification factor and $f_{\text {ion }} \equiv n_{n} /\left(n_{n}+n_{i}\right)$ is the ionization fraction. $n_{n}$ and $n_{i}$ is the neutral particle density and the ionized particle density, respectively. However, we note that ion-neutral collisions suppress Alfvén wave [56, 74] at $v_{s} \lesssim 120 \mathrm{~km} \mathrm{~s}^{-1}$. In this paper, we take Equation 9 as the fiducial value for the downstream magnetic field.

The diffusive shock acceleration timescale can be written as

$$
t_{\mathrm{acc}} \approx \frac{10}{3} \frac{\eta c r_{g}}{v_{s}^{2}} \sim 1.3 \times 10^{6} Z^{-1} n_{\mathrm{gas}, 0.5}^{-1 / 2} v_{s, 7.1}^{-2} E_{\mathrm{cr}, 14} \mathrm{yr},
$$

where $r_{g}=E_{\mathrm{cr}} / Z e B_{d}$ is the gyroradius and $\eta \geq 1$. For simplicity, we take $\eta=1$ in this paper, i.e. the Bohm limit, since ULXs are known to be associated with star forming regions [e.g. 33, 72, 81] which would amplify the upstream turbulence. Such an efficient acceleration is possibly seen in a Galactic supernova remnant by assuming a simple diffusive shock acceleration model [83], although $\eta$ can be different from unity by considering escape-limited acceleration $[34,68]$ or stochastic acceleration [26].

The attainable maximum cosmic-ray energy is given by $t_{\mathrm{acc}}=$ $t_{\text {dyn }}$ (See Equation 5 and Equation 10):

$$
\begin{aligned}
E_{\mathrm{cr}, \text { max }} & =\frac{3 Z e B_{d} R_{\mathrm{b}} v_{s}}{10 \eta c} \\
& \sim 1.3 \times 10^{14} \eta_{0} n_{\text {gas }, 0.5}^{1 / 2} v_{s, 7.1} R_{\mathrm{b}, 20.8} \mathrm{eV},
\end{aligned}
$$

$\mathrm{TeV}$ cosmic rays can be affordable from the extragalactic X-ray source nebulae. If the Alfvén-wave turbulence is fully generated, it will reach to $\sim 1 \mathrm{PeV}$.

The ionization state in the shock downstream strongly depends on its velocity $[36,56,74]$. If neutral particle exists, i.e. $v_{s} \lesssim 120 \mathrm{~km} \mathrm{~s}^{-1}$, ion-neutral collisions will lead damping of the magnetic turbulence in the shock precursor and those collisions will hamper acceleration of particles at the highest energies. The expected break energy can be estimated as [60, and references therein]

$$
E_{\text {cr,br }} \sim 1.9 \times 10^{9} T_{u, 4}^{-0.4} B_{u,-6}^{2}\left(1-f_{\text {ion }}\right)^{-1} f_{\text {ion }}^{-1 / 2} n_{\text {gas }, 0.5}^{-3 / 2} \mathrm{eV} .
$$

Thus, at $v_{s} \lesssim 120 \mathrm{~km} \mathrm{~s}^{-1}$, the particle spectrum will have a break at $\sim 10 \mathrm{GeV}$ and become steeper by one power above the break. The ion fraction $f_{\text {ion }}$ is estimated according to the steady-state model by Hollenbach and McKee [36]. 
Table 1: Observed Properties of Nebulae Associated with Extragalactic Compact X-ray Objects

\begin{tabular}{lcccc}
\hline Object & $d$ & $L_{X}$ & $R_{b}$ & $v_{\mathrm{s}}$ \\
\hline & $(\mathrm{Mpc})$ & $\left(\mathrm{erg} \mathrm{s}^{-1}\right)$ & $(\mathrm{pc})$ & $\left(\mathrm{km} \mathrm{s}^{-1}\right)$ \\
\hline Microquasar S26 & 3.9 & $6.2 \times 10^{36}$ & 200 & 250 \\
ULX IC 342 X-1 & 3.9 & $1.6 \times 10^{40}$ & 200 & 100 \\
HMXB IC 10 X-1 & 0.79 & $1.5 \times 10^{38}$ & 170 & 80 \\
\hline
\end{tabular}

Table 2: The table data are taken from Pakull et al. [70, for S26], Allen et al. [10], Cseh et al. [20, for IC 342 X-1], and Lozinskaya and Moiseev [58, for IC $10 \mathrm{X}-1]$. The data for the time-averaged X-ray luminosity are from Cseh et al. [20].

Let us consider energy loss processes for cosmic rays. The energy loss timescale due to $p p$ interactions can be estimated as

$$
t_{p p} \approx \frac{1}{\kappa_{p p} 4 n_{\mathrm{gas}} \sigma_{p p} c} \sim 6.0 \times 10^{6} n_{\mathrm{gas}, 0.5}^{-1} \mathrm{yr}
$$

where the cross section $\sigma_{p p} \sim 3 \times 10^{-26} \mathrm{~cm}^{2}$ for $10^{10-12} \mathrm{eV}$ $[46,48]$ and the inelasticity $\kappa_{p p} \approx 0.5$. The factor of 4 in front of $n_{\text {gas }}$ is due to compression in the downstream. We note that the $p p$ cross section depends logarithmically on the proton energy.

By considering the steady-state for simplicity, the hadronuclear interaction efficiency is estimated as

$$
f_{\mathrm{pp}}=\frac{t_{\mathrm{dyn}}}{t_{\mathrm{pp}}} \sim 0.28 R_{\mathrm{b}, 20.8} v_{s, 7.1}^{-1} n_{\mathrm{gas}, 0.5}
$$

In hadronuclear interactions, charged and neutral pions are generated at the ratio of $\pi^{+}: \pi^{0} \approx 2: 1$. Gamma rays are produced by the decay of neutral pions as $\pi^{0} \rightarrow 2 \gamma$.

If we neglect ion-neutral collisions, gamma-ray flux from an expanding nebula can be estimated as

$$
\begin{aligned}
E_{\gamma}^{2} \frac{d N_{\gamma}}{d E_{\gamma}} & \approx \frac{1}{3} \frac{\epsilon_{\mathrm{M}} \xi_{\mathrm{cr}} P_{\mathrm{kin}} \min \left[1, f_{p p}\right]}{4 \pi d^{2} \mathrm{C}} \\
& \sim 1.1 \times 10^{-13} \mathrm{erg} \mathrm{cm}^{-2} \mathrm{~s}^{-1}\left(\frac{C}{12}\right)^{-1}\left(\frac{d}{2 \mathrm{Mpc}}\right)^{-2} \\
& \times \xi_{\mathrm{cr},-1} R_{\mathrm{b}, 20.8}^{3} v_{s, 7.1}^{2} n_{\mathrm{gas}, 0.5}^{2}
\end{aligned}
$$

where $\xi_{\text {cr }}$ is the cosmic-ray acceleration efficiency and $C=$ $\ln \left(E_{p, \text { max }} / E_{p, \text { min }}\right)$. We set $E_{p, \text { max }}=10^{14} \mathrm{eV}$ and $E_{p, \text { min }}=10^{9} \mathrm{eV}$. We set the nuclear enhancement factor $\epsilon_{M}=1.85$ as in our Galaxy [e.g., 65, 78]. ULXs are known to be hosted by lowmetallicity galaxies at the metallicity of $Z \lesssim 1 / 2 Z_{\odot}$ [61]. Thus, $\epsilon_{M}$ would be lower than that in our galaxy. However, it is known that a galaxy is not chemically homogeneous and can have metallicity dispersion by a factor of 10 in a galaxy [e.g. 67, 73]. We note that an X-ray measurement of the ULX NGC 1313 X-1 revealed that the local oxygen abundance is $\sim 50 \%$ of the solar value using the low energy X-ray absorption feature [64].

We can also evaluate the expected neutrino flux per flavor as $E_{\gamma} d N_{\gamma} / d E_{\gamma} \approx 2 \times E_{\nu} d N_{v} / d E_{v}\left(E_{v}=0.5 E_{\gamma}\right)$, or

$$
\begin{aligned}
E_{v}^{2} \frac{d N_{v}}{d E_{v}} & \sim 5.6 \times 10^{-14} \mathrm{erg} \mathrm{cm}^{-2} \mathrm{~s}^{-1}\left(\frac{C}{12}\right)^{-1}\left(\frac{d}{2 \mathrm{Mpc}}\right)^{-2} \\
& \times \xi_{\mathrm{cr},-1} R_{\mathrm{b}, 20.8}^{3} v_{s, 7.1}^{2} n_{\mathrm{gas}, 0.5}^{2} .
\end{aligned}
$$

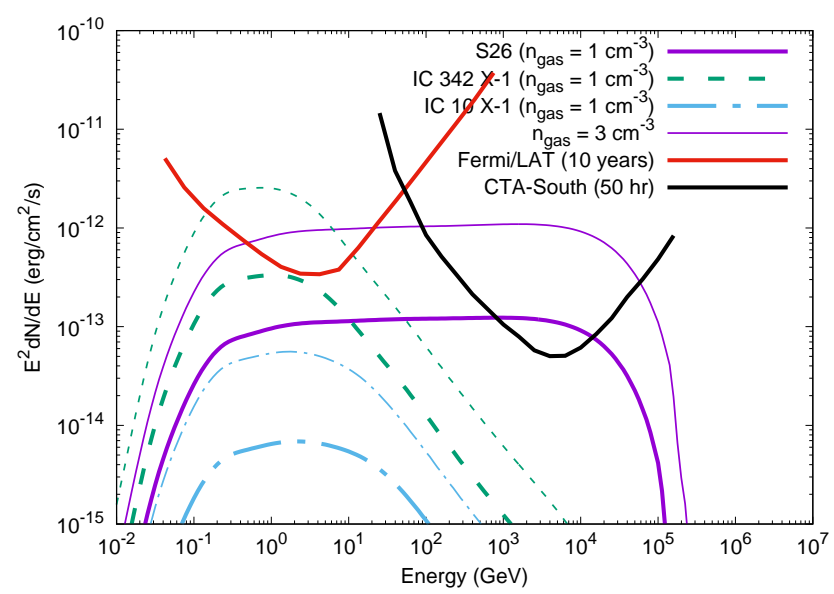

Figure 1: Expected gamma-ray spectra from nebulae associated with microquasar S26, ULX IC 342 X-I, and HMXB IC 10 X-I shown by solid, dashed, and dot-dashed curve, respectively. Thick curves represent the case with $n_{\text {gas }}=1 \mathrm{~cm}^{-3}$, while thin curves show $n_{\text {gas }}=3 \mathrm{~cm}^{-3}$. The sensitivity of Fermi/LAT and CTA in the southern site is shown for $10 \mathrm{yr}$ and $50 \mathrm{hr}$ integration, respectively.

Figure 1 shows the expected gamma-ray signals from nebulae hosting compact X-ray sources in the nearby Universe together with the Fermi/LAT ${ }^{3}$ and CTA-South ${ }^{4}$ sensitivities for $^{-}$ 10 -yr integration and a $50 \mathrm{hr}$ observation, respectively. We select representative sources from Cseh et al. [20] which include microquasar S26 [70], ULX IC 342-X-1 [10, 20], and highmass X-ray binary (HMXB) IC 10-X-1 [58]. The parameters are summarized in Table 1. For the calculation of the $p p$ interaction, we follow the prescription provided by Kamae et al. [48] setting the fraction of the energy received in the pion as 0.17 [52]. For the gas density, we consider two cases with $n_{\text {gas }}=1 \mathrm{~cm}^{-3}$ and $3 \mathrm{~cm}^{-3}$. Here, ULXs are known to be associated with star forming regions [e.g. 33, 72, 81] and the gas density in the $\mathrm{H}$ II region can be as high as $\sim 10 \mathrm{~cm}^{-3}$ for the size of $\sim 100 \mathrm{pc}$ [37].

The nebula associated with the nearby microquasar S26 shows a flat spectrum in $E^{2} d N / d E$ extending to $\sim 100 \mathrm{TeV}$ and is detectable even for $n_{\text {gas }}=1 \mathrm{~cm}^{-3}$. Thus, S26 would be a good target for future CTA observation. On the contrary, the nebulae associated with IC $342 \mathrm{X}-1$ and IC $10 \mathrm{X}-1$ would have soft gamma-ray spectra at $\gtrsim 10 \mathrm{GeV}$ due to ion-neutral collisions, as the expansion velocity is slower than $120 \mathrm{~km} \mathrm{~s}^{-1}$. Such slowly expanding nebulae would be difficult to be observed by CTA.

Figure 2 shows the expected neutrino signal per flavour from the nebula surrounding S26 together with the IceCube sensitivity [2]. If the gas density is as high as $n_{\text {gas }}=3 \mathrm{~cm}^{-3}$, an order of magnitude sensitive neutrino detectors will be able to see the signal.

Bordas et al. [15] reported the marginal detection of gamma rays from the direction of the Galactic microquasar SS 433

\footnotetext{
${ }^{3}$ https://www.slac.stanford.edu/exp/glast/groups/canda/lat_Perform

${ }^{4}$ https://portal.cta-observatory.org/Pages/CTA-Performance.aspx
} 


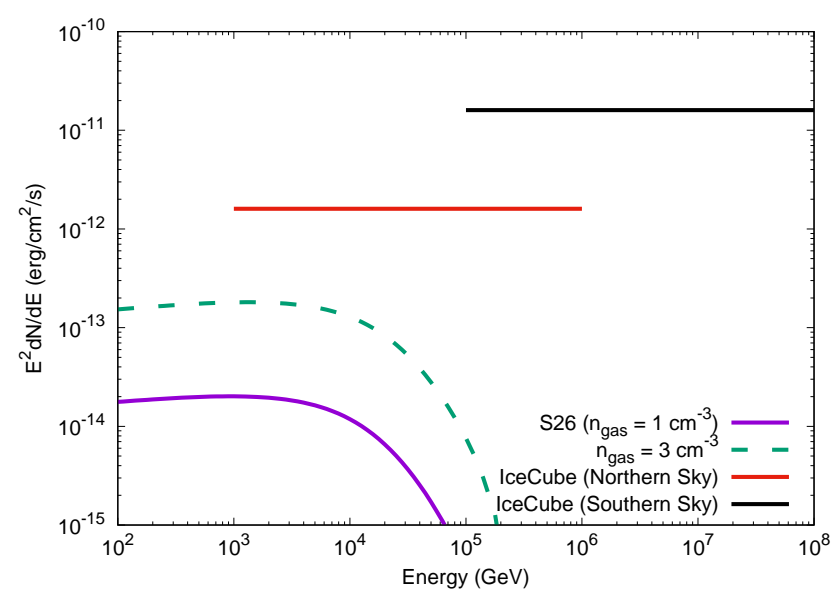

Figure 2: Expected neutrino spectrum per flavor from the nebula associated with microquasar S26. The thick and dashed curve represents the case with $n_{\text {gas }}=1 \mathrm{~cm}^{-3}$ and $3 \mathrm{~cm}^{-3}$, respectively. The sensitivities of IceCube for the northern sky and the southern sky [2] are also shown, respectively.

and the related supernova remnant W50 using Fermi. These gamma-ray signals may arise from the particle acceleration in the associated nebula. However, the shell expansion velocity is known to be $16-40 \mathrm{~km} \mathrm{~s}^{-1}$ [57] which would be inefficient to accelerate particles in the expanding shell.

Electrons are also expected to be accelerated together with protons. To evaluate the leptonic contribution, we need an electron-to-proton ratio $K_{e p}$, where $K_{e p}$ is defined as the ratio of electron spectrum $d N_{e} / d p$ and proton spectrum $d N_{p} / d p$ at $p=1 \mathrm{GeV} \mathrm{c}^{-1}$. In this paper, we take $K_{e p}=0.01$ as the fiducial value, since this value is favored for Galactic SNRs in the hadronuclear scenario [5]. The expected radio synchrotron flux by electrons is given by $f_{e, \text { syn }} \approx c \sigma_{T} U_{B} \gamma_{e, s}^{3} N_{e}\left(\gamma_{e, s}\right) / 6 \pi d^{2}$ [22]. Thus, the radio flux by primary electrons is approximately given as

$$
\begin{aligned}
E_{\gamma}^{2} \frac{d N_{\gamma}}{d E_{\gamma}} & \sim 1.2 \times 10^{-16} \mathrm{erg} \mathrm{cm}^{-2} \mathrm{~s}^{-1}\left(\frac{C}{12}\right)^{-1}\left(\frac{d}{2 \mathrm{Mpc}}\right)^{-2} \\
& \times K_{e p,-2} \xi_{\mathrm{cr},-1} R_{\mathrm{b}, 20.8}^{3} v_{s, 7.1}^{2} n_{\mathrm{gas}, 0}^{7 / 4} v_{s, 9.7}^{1 / 2}
\end{aligned}
$$

where $v_{s}$ is a frequency of the synchrotron component. By adopting the parameters for S26 and assuming $K_{e p}=0.01$, we get $\sim 2.6 \mathrm{mJy}$ and $\sim 2.0 \mathrm{mJy}$ at 5.5 and $9.0 \mathrm{GHz}$, respectively, with $n_{\text {gas }}=3 \mathrm{~cm}^{-3}$ which are consistent with the measured radio flux of S26 of $2.1 \mathrm{mJy}$ at $5.5 \mathrm{GHz}$ and $1.6 \mathrm{mJy}$ at $9.0 \mathrm{GHz}$ [77]. Thus, determination of gamma-ray spectrum will be also useful to understand the origin of the radio emission and to constrain the environment of the nebula. If the downstream magnetic field is amplified by Alfvén-wave turbulence, the required $K_{e p}$ becomes $7 \times 10^{-5}$ to be consistent with the radio observations.

Here, the inverse Compton (IC) flux by electrons scattering the cosmic microwave background (CMB) photons is given as $f_{\mathrm{IC}} \approx c \sigma_{T} \gamma_{e, s}^{3} N_{e}\left(\gamma_{e, s}\right) u_{\mathrm{CMB}} / 6 \pi d^{2}$, where a monochromatic radiation field assumed for the CMB and we assume the Thomson regime [22]. The IC flux is approximately

$$
\begin{aligned}
E_{\gamma}^{2} \frac{d N_{\gamma}}{d E_{\gamma}} & \sim 1.4 \times 10^{-13} \mathrm{erg} \mathrm{cm}^{-2} \mathrm{~s}^{-1}\left(\frac{C}{12}\right)^{-1}\left(\frac{d}{2 \mathrm{Mpc}}\right)^{-2} \\
& \times K_{e p,-2} \xi_{\mathrm{cr},-1} R_{\mathrm{b}, 20.8}^{3} v_{s, 7.1}^{2} n_{\mathrm{gas}, 0}^{7 / 4} E_{\gamma, 12}^{1 / 2}
\end{aligned}
$$

Therefore, the IC contribution from primary electrons would become important at $E_{\gamma} \sim 1 \mathrm{TeV}$ if $K_{e p} \gtrsim 0.01$. Since secondary electrons carry only $\sim 0.8 \%$ of the primary proton energy, their contribution would be less important. If the local optical or infrared (IR) photon energy density is comparable to the CMB energy density, the total IC contribution would be enhanced. The local nebular luminosity of S26 is $\sim 5 \times 10^{39} \mathrm{erg} \mathrm{s}^{-1}$ [23] corresponding to the photon energy density of $\sim 0.020 \mathrm{eV} \mathrm{cm}^{-3}$. Thus, the IC flux for $\mathrm{S} 26$ would not be significantly enhanced even if we take into account local photon fields other than CMB photons.

\section{Discussions}

\subsection{Spectral Index of Accelerated Cosmic Rays}

In this paper, we assume the spectral index of 2 for accelerated particles. This assumption would be violated by various possible mechanisms. As the source S26 expands at the velocity of $250 \mathrm{~km} \mathrm{~s}^{-1}$ corresponding to the sonic and Alfvénic mach number of 17 and 35, respectively, we can expect the compression ratio $r \sim 4$ for the sources S26 in NGC 7793. However, for sources having lower expansion velocity, the compression ratio would become smaller resulting in softer spectra [e.g. 17]. In addition to this effect, the ion-neutral collision effect $[36,56,74]$ and the effect of finite velocity of the counter streaming magnetic turbulence in front of the shock [e.g. 16, 55] would steepen the cosmic-ray spectral index. On the contrary, in the radiative region, we expect a larger compression ratio than $r=4$. The size of the radiative region is $l_{\text {rad }} \approx v_{s} t_{\text {rad }} / 4 \sim$ $4.3 \times 10^{-2} n_{\text {gas }, 0.5} v_{s, 7.1}^{22 / 5} \mathrm{pc}$, while the size of the acceleration region is $l_{\mathrm{acc}} \approx v_{s} t_{\mathrm{acc}} \sim 4.8 \times 10^{-2} Z^{-1} n_{\mathrm{gas}, 0.5}^{-1 / 2} v_{s, 7.1}^{-1} E_{\mathrm{cr}, 10.5} \mathrm{pc}$. Thus, the spectral index would become harder than 2 above $\sim 30 \mathrm{GeV}$ and more photons would be detectable at the $\mathrm{TeV}$ band.

\subsection{Cosmic Gamma-ray and Neutrino Background Radiation}

The cosmic GeV gamma-ray background radiation is known to be composed of blazars [e.g. 9, 43], radio galaxies [39], and star forming galaxies [6]. However, there is still an unresolved component at the flux level of $7.2 \times 10^{-6} \mathrm{~cm}^{-2} \mathrm{~s}^{-1} \mathrm{sr}^{-1}$ above $0.1 \mathrm{GeV}$ [4]. If nebulae associated with microquasars and ULXs emit gamma rays, they would also contribute to the unresolved cosmic gamma-ray background radiation.

The local X-ray luminosity functions of ULXs are well studied in literature [e.g. 32, 62, 80, 85]. The local number density of ULXs is $n_{\mathrm{ULX}} \sim 1.5 \times 10^{-2} \mathrm{Mpc}^{-3}$ [80]. Then, the cosmic gamma-ray background flux from ULX/microquasar nebu- 
lae can be estimated as

$$
\begin{aligned}
E_{\gamma}^{2} \frac{d I_{\gamma}}{d E_{\gamma}} & \approx \frac{1}{3} \frac{c t_{H} \xi_{z}}{4 \pi} \frac{\epsilon_{\mathrm{M}} \xi_{\mathrm{cr}} P_{\mathrm{kin}} n_{\mathrm{ULX}} \min \left[1, f_{p p}\right]}{C} \\
& \sim 5.2 \times 10^{-6} \mathrm{MeV} \mathrm{cm}^{-2} \mathrm{~s}^{-1} \mathrm{sr}^{-1}\left(\frac{\xi_{z}}{3}\right)^{-1}\left(\frac{C}{12}\right)^{-1} \\
& \times \xi_{\mathrm{cr},-1} R_{\mathrm{b}, 20.8}^{3} v_{s, 7.1}^{2} n_{\mathrm{gas}, 0.5}^{2} n_{\mathrm{ULX},-1.8},
\end{aligned}
$$

where $t_{H} \sim 13.8 \mathrm{Gyr}$ is the Hubble timescale assuming $H_{0}=$ $67.8 \mathrm{~km} \mathrm{~s}^{-1} \mathrm{Mpc}^{-1}$ and $\Omega_{M}=0.308$ [71]. $\xi_{z}$ is a factor accounting cosmological evolution of the source. We assume $v_{s} \geq 120 \mathrm{~km} \mathrm{~s}^{-1}$ for all sources. Therefore, ULX/microquasar nebulae would contribute $5.2 \times 10^{-7} \mathrm{~cm}^{-2} \mathrm{~s}^{-1} \mathrm{sr}^{-1}$ above $0.1 \mathrm{GeV}$ ( $\sim 7 \%$ of the unresolved background flux).

The cosmic neutrino background flux would become

$$
\begin{aligned}
E_{v}^{2} \frac{d I_{v}}{d E_{v}} & \sim 2.6 \times 10^{-9} \mathrm{GeV} \mathrm{cm}^{-2} \mathrm{~s}^{-1} \mathrm{sr}^{-1}\left(\frac{\xi_{z}}{3}\right)^{-1}\left(\frac{C}{12}\right)^{-1} \\
& \times \xi_{\mathrm{cr},-1} R_{\mathrm{b}, 20.8}^{3} v_{s, 7.1}^{2} n_{\mathrm{gas}, 0.5}^{2} n_{\mathrm{ULX},-1.8}
\end{aligned}
$$

where we note that the neutrino spectra from ULX nebulae would have a cutoff at several tens TeV (see Equation 12). Due to this cutoff, the nebulae would not contribute to the IceCube detected neutrino fluxes [1]. However, if Alfvén-wave turbulence at the shock is fully generated, sub-PeV neutrinos can be affordable.

\subsection{Gamma-ray Emission and Attenuation in Host Galaxies}

The total IR luminosity of NGC 7793 which hosts the microquasar S26 is $2.07 \times 10^{9} L_{\odot}$ [29]. Adopting the scaling relation between gamma-ray and IR luminosities [6], we have $L_{\gamma} \sim 3.8 \times 10^{38} \mathrm{erg} \mathrm{s}^{-1}$ for galactic diffuse emission leading $E_{\gamma} d N_{\gamma} / d E_{\gamma} \sim 2.2 \times 10^{-13} \mathrm{erg} \mathrm{cm}^{-2} \mathrm{~s}^{-1}$ which is comparable to expected flux from S26 assuming $n_{\text {gas }}=1 \mathrm{~cm}^{-3}$. Here, the angular size of NGC 7793 is $9^{\prime} 3 \times 6^{\prime} 3$ and S26 locates at the outskirt region of the galaxy ( $\sim 3 \mathrm{arcmin}$ away from the nucleus). Given the CTA angular resolution of $\lesssim 3 \operatorname{arcmin}$ at $\gtrsim 1 \mathrm{TeV}$, gamma-ray signals from S26 can be distinguished from gamma rays due to galactic cosmic rays which would be bright in the star forming nucleus region.

It is well-known that gamma rays propagating the intergalactic space are attenuated by the cosmic optical/IR background radiation field via the pair production process [31, 45, 79]. Since we are considering the local universe $\lesssim 10 \mathrm{Mpc}$, the gammaray attenuation by the cosmic optical/IR background is negligible [e.g. 41]. However, if ULXs and microquasars are formed in the star forming regions, internal gamma-ray attenuation by the interstellar radiation fields will suppress gamma-ray flux above $10 \mathrm{TeV}$ [see e.g., 40].

\subsection{ULX pulsars}

Recently, some ULXs are discovered to be hosted by accreting pulsars [11, 28, 44]. Physical properties of ULX pulsars are still open questions. Magnetic fields of M82 X-2 are ranging $10^{9} \mathrm{G} \lesssim B \lesssim 10^{14} \mathrm{G}$ in literatures [18, 25, 49, 53, 54, 66, 82].

If we assume that X-ray emissions from ULX pulsars are generated at accreting column [e.g. 50], the accretion shock may accelerate particles near the polar cap region of the pulsars. However, $\gtrsim 10 \mathrm{GeV}$ gamma rays from the polar cap region emission will be attenuated due to one-photon pair creation. The threshold magnetic field strength is $B \gtrsim 0.1 B_{Q}$ $[21,35]$, where $B_{Q}=m_{e}^{2} c^{3} / e=4.4 \times 10^{13} \mathrm{G}$. Therefore, strong gamma-ray emission would not be expected for high magnetic field ULX pulsars.

\section{Conclusions}

In the coming decade, CTA will deepen our understandings of high energy astrophysical phenomena. In this paper, we investigate high energy signals from nebulae associated with microquasars and ULXs in the nearby Universe whose available power is $\gtrsim 10^{39} \mathrm{erg} \mathrm{s}^{-1}$.

Although gamma rays from host galaxy itself exist, CTA's sensitivity and angular resolution allow us to detect and resolve powerful nebulae associated with microquasars and ULXs. For example, the nebula associated with the microquasar S26 in NGC 7793 [70] will be a good target whose expected gammaray flux is $\sim 1 \times 10^{-13}-1 \times 10^{-12} \mathrm{erg} \mathrm{cm}^{-2} \mathrm{~s}^{-1}$ extending to several tens $\mathrm{TeV}$ with a photon index of $\sim 2$. At $\gtrsim 1 \mathrm{TeV}$, the IC contribution would become important if $K_{e p} \gtrsim 0.01$. We note that the spectral index of cosmic rays would be harder than 2 above $\sim 30 \mathrm{GeV}$ since it is the radiative shock. We also found that the expected synchrotron radiation flux from primary electrons is consistent with the measured radio flux. If all microquasars and ULXs are associated with powerful expanding nebulae, they would make $\sim 7 \%$ of the unresolved cosmic gammaray background flux. However, they would not significantly contribute to the TeV-PeV neutrino background flux reported by IceCube due to cutoffs at several tens $\mathrm{TeV}$ in neutrino spectra. If the expansion velocity is $v_{s} \lesssim 120 \mathrm{~km} \mathrm{~s}^{-1}$, ion-neutral collisions will suppress efficient particle acceleration and result in fainter TeV gamma-ray flux above $\sim 10 \mathrm{GeV}$.

\section{Acknowledgements:}

The authors thank Chris Done for useful comments and discussions. YI is supported by the JAXA international top young fellowship and JSPS KAKENHI Grant Number 420 JP16K13813.

\section{References}

[1] Aartsen, M.G., Ackermann, M., Adams, J., et al., 2014a. Observation of High-Energy Astrophysical Neutrinos in Three Years of IceCube Data. Physical Review Letters 113, 101101. doi:10.1103/PhysRevLett.113.101101, arXiv:1405.5303.

[2] Aartsen, M.G., Ackermann, M., Adams, J., et al., 2014b. Searches for Extended and Point-like Neutrino Sources with Four Years of IceCube Data. ApJ 796, 109. doi:10.1088/0004-637X/796/2/109, arXiv: 1406.6757.

[3] Acero, F., Bamba, A., Casanova, S., et al., 2013. Gammaray signatures of cosmic ray acceleration, propagation, and confinement in the era of CTA. Astroparticle Physics 43, 276-286. doi:10.1016/j.astropartphys.2012.05.024, arXiv: 1209. 0582.

[4] Ackermann, M., Ajello, M., Albert, A., et al., 2015. The Spectrum of Isotropic Diffuse Gamma-Ray Emission between $100 \mathrm{MeV}$ and $820 \mathrm{GeV}$. ApJ 799, 86. doi:10.1088/0004-637X/799/1/86, arXiv: 1410.3696 . 
[5] Ackermann, M., Ajello, M., Allafort, A., et al., 2013. Detection of the Characteristic Pion-Decay Signature in Supernova Remnants. Science 339, 807-811. doi:10.1126/science.1231160, arXiv: 1302.3307.

[6] Ackermann, M., Ajello, M., Allafort, A., et al., 2012. GeV Observations of Star-forming Galaxies with the Fermi Large Area Telescope. ApJ 755, 164. doi:10.1088/0004-637X/755/2/164, arXiv:1206.1346.

[7] Ackermann, M., Ajello, M., Atwood, W.B., et al., 2016. 2FHL: The Second Catalog of Hard Fermi-LAT Sources. ApJS 222, 5. doi:10.3847/0067-0049/222/1/5, arXiv: 1508.04449.

[8] Actis, M., Agnetta, G., Aharonian, F., et al., 2011. Design concepts for the Cherenkov Telescope Array CTA: an advanced facility for groundbased high-energy gamma-ray astronomy. Experimental Astronomy 32, 193-316. doi:10.1007/s10686-011-9247-0, arXiv:1008.3703.

[9] Ajello, M., Gasparrini, D., Sánchez-Conde, M., et al., 2015. The Origin of the Extragalactic Gamma-Ray Background and Implications for Dark Matter Annihilation. ApJL 800, L27. doi:10.1088/2041-8205/800/2/L27, arXiv: 1501.05301.

[10] Allen, M.G., Groves, B.A., Dopita, M.A., Sutherland, R.S., Kewley, L.J., 2008. The MAPPINGS III Library of Fast Radiative Shock Models. ApJS 178, 20-55. doi:10.1086/589652, arXiv:0805.0204.

[11] Bachetti, M., Harrison, F.A., Walton, D.J., et al., 2014. An ultraluminous X-ray source powered by an accreting neutron star. Nature 514, 202-204. doi:10.1038/nature13791, arXiv: 1410.3590.

[12] Bell, A.R., 1978a. The acceleration of cosmic rays in shock fronts. I. MNRAS 182, 147-156. doi:10.1093/mnras/182.2.147.

[13] Bell, A.R., 1978b. The acceleration of cosmic rays in shock fronts. II MNRAS 182, 443-455. doi:10.1093/mnras/182.3.443.

[14] Bell, A.R., 2004. Turbulent amplification of magnetic field and diffusive shock acceleration of cosmic rays. MNRAS 353, 550-558. doi:10.1111/j.1365-2966.2004.08097.x.

[15] Bordas, P., Yang, R., Kafexhiu, E., Aharonian, F., 2015. Detection of Persistent Gamma-Ray Emission Toward SS433/W50. ApJL 807, L8. doi:10.1088/2041-8205/807/1/L8, arXiv: 1411.7413.

[16] Bykov, A.M., Ellison, D.C., Osipov, S.M., Vladimirov, A.E., 2014. Magnetic Field Amplification in Nonlinear Diffusive Shock Acceleration Including Resonant and Non-resonant Cosmic-Ray Driven Instabilities. ApJ 789, 137. doi:10.1088/0004-637X/789/2/137, arXiv: 1406.0084

[17] Caprioli, D., Spitkovsky, A., 2014. Simulations of Ion Acceleration at Non-relativistic Shocks. I. Acceleration Efficiency. ApJ 783, 91. doi:10.1088/0004-637X/783/2/91, arXiv : 1310.2943.

[18] Chen, W.C., 2016. Constraining the dipolar magnetic field of M82 X-2 by the accretion model. ArXiv e-prints arXiv: 1609.05706

[19] Crutcher, R.M., 1999. Magnetic Fields in Molecular Clouds: Observations Confront Theory. ApJ 520, 706-713. doi:10.1086/307483.

[20] Cseh, D., Corbel, S., Kaaret, P., et al., 2012. Black Hole Powered Nebulae and a Case Study of the Ultraluminous X-Ray Source IC 342 X-1. ApJ 749, 17. doi:10.1088/0004-637X/749/1/17, arXiv: 1201.4473

[21] Daugherty, J.K., Harding, A.K., 1982. Electromagnetic cascades in pulsars. ApJ 252, 337-347. doi:10.1086/159561.

[22] Dermer, C.D., Menon, G., 2009. High Energy Radiation from Black Holes: Gamma Rays, Cosmic Rays, and Neutrinos

[23] Dopita, M.A., Payne, J.L., Filipović, M.D., Pannuti, T.G., 2012. The physical parameters of the microquasar S26 in the Sculptor Group galaxy NGC 7793. MNRAS 427, 956-967. doi:10.1111/j.1365-2966.2012.21947.x, arXiv:1208.3500.

[24] Draine, B.T., 2011. Physics of the Interstellar and Intergalactic Medium.

[25] Ekşi, K.Y., Andaç, İ.C., Çıkıntoğlu, S., Gençali, A.A., Güngör, C., Öztekin, F., 2015. The ultraluminous X-ray source NuSTAR J095551+6940.8: a magnetar in a high-mass X-ray binary. MNRAS 448, L40-L42. doi:10.1093/mnrasl/slu199, arXiv: 1410.5205.

[26] Fan, Z., Liu, S., Fryer, C.L., 2010. Stochastic electron acceleration in the TeV supernova remnant RX J1713.7-3946: the high-energy cut-off. MNRAS 406, 1337-1349. doi:10.1111/j.1365-2966.2010.16767.x, arXiv:0909.3349.

[27] Feng, H., Soria, R., 2011. Ultraluminous X-ray sources in the Chandra and XMM-Newton era. New Astronomy Review 55, 166-183. doi:10.1016/j.newar.2011.08.002, arXiv:1109.1610.

[28] Fuerst, F., Walton, D.J., Harrison, F.A., et al., 2016. Discovery of coherent pulsations from the Ultraluminous X-ray Source NGC 7793 P13. ArXiv e-prints arXiv: 1609.07129.

[29] Galametz, M., Kennicutt, R.C., Calzetti, D., et al., 2013. Calibration of the total infrared luminosity of nearby galaxies from Spitzer and Herschel bands. MNRAS 431, 1956-1986. doi:10.1093/mnras/stt313, arXiv: 1302.4363

[30] Gladstone, J.C., Roberts, T.P., Done, C., $2009 . \quad$ The ultraluminous state. MNRAS 397, 1836-1851. doi:10.1111/j.1365-2966.2009.15123.x, arXiv:0905.4076.

[31] Gould, R.J., Schréder, G., 1966. Opacity of the Universe to High-Energy Photons. Physical Review Letters 16, 252-254. doi:10.1103/PhysRevLett.16.252.

[32] Grimm, H.J., Gilfanov, M., Sunyaev, R., 2003. High-mass X-ray binaries as a star formation rate indicator in distant galaxies. MNRAS 339, 793-809. doi:10.1046/j.1365-8711.2003.06224.x, arXiv: astro-ph/0205371.

[33] Grisé, F., Kaaret, P., Pakull, M.W., Motch, C., 2011. Optical Properties of the Ultraluminous X-Ray Source Holmberg IX X-1 and Its Stellar Environment. ApJ 734, 23. doi:10.1088/0004-637X/734/1/23, arXiv:1104.5523.

[34] H. E. S. S. Collaboration, Abdalla, H., Abdalla, H., et al., 2016. H.E.S.S. observations of RX J1713.7-3946 with improved angular and spectral resolution; evidence for gamma-ray emission extending beyond the X-ray emitting shell. ArXiv e-prints arXiv: 1609.08671.

[35] Harding, A.K., Lai, D., 2006. Physics of strongly magnetized neutron stars. Reports on Progress in Physics 69, 2631-2708. doi:10.1088/0034-4885/69/9/R03, arXiv : astro-ph/0606674

[36] Hollenbach, D., McKee, C.F., 1989. Molecule formation and infrared emission in fast interstellar shocks. III - Results for J shocks in molecular clouds. ApJ 342, 306-336. doi:10.1086/167595

[37] Hunt, L.K., Hirashita, H., 2009. The size-density relation of extragalactic $\mathrm{H}$ II regions. A\&A 507, 1327-1343. doi:10.1051/0004-6361/200912020, arXiv:0910.2804.

[38] Inoue, S., Granot, J., O’Brien, P.T., et al., 2013a. Gamma-ray burst science in the era of the Cherenkov Telescope Array. Astroparticle Physics 43, 252-275. doi:10.1016/j . astropartphys. 2013.01.004, arXiv: 1301.3014

[39] Inoue, Y., 2011a. Contribution of Gamma-Ray-loud Radio Galaxies' Core Emissions to the Cosmic MeV and GeV Gamma-Ray Background Radiation. ApJ 733, 66. doi:10.1088/0004-637X/733/1/66, arXiv: 1103.3946

[40] Inoue, Y., 2011b. High Energy Gamma-ray Absorption and Cascade Emission in Nearby Starburst Galaxies. ApJ 728, 11. doi:10.1088/0004-637X/728/1/11, arXiv: 1011.6511.

[41] Inoue, Y., Inoue, S., Kobayashi, M.A.R., Makiya, R., Niino, Y., Totani, T., 2013b. Extragalactic Background Light from Hierarchical Galaxy Formation: Gamma-Ray Attenuation up to the Epoch of Cosmic Reionization and the First Stars. ApJ 768, 197. doi:10.1088/0004-637X/768/2/197, arXiv: 1212.1683.

[42] Inoue, Y., Tanaka, Y.T., Isobe, N., 2016. Binary black hole merger rates inferred from luminosity function of ultra-luminous X-ray sources. MNRAS 461, 4329-4334. doi:10.1093/mnras/stw1637, arXiv: 1602.05554.

[43] Inoue, Y., Totani, T., 2009. The Blazar Sequence and the Cosmic Gamma-ray Background Radiation in the Fermi Era. ApJ 702, 523-536. doi:10.1088/0004-637X/702/1/523, arXiv:0810.3580.

[44] Israel, G.L., Belfiore, A., Stella, L., et al., 2016. ULX-1 in NGC5907: how bright can an accreting pulsar shine? ArXiv e-prints arXiv: 1609.07375

[45] Jelley, J.V., 1966. High-Energy $\gamma$-Ray Absorption in Space by a 3.5degK Microwave Field. Physical Review Letters 16, 479-481. doi:10.1103/PhysRevLett.16.479

[46] Kafexhiu, E., Aharonian, F., Taylor, A.M., Vila, G.S., 2014. Parametrization of gamma-ray production cross sections for $p$ interactions in a broad proton energy range from the kinematic threshold to $\mathrm{PeV}$ energies. Phys. Rev. D 90, 123014. doi:10.1103/PhysRevD.90.123014, arXiv: 1406.7369

[47] Kaiser, C.R., Alexander, P., 1997. A self-similar model for extragalactic radio sources. MNRAS 286, 215-222. doi:10.1093/mnras/286.1.215.

[48] Kamae, T., Karlsson, N., Mizuno, T., Abe, T., Koi, T., 2006. Parameterization of $\gamma, \mathrm{e}^{+/-}$, and Neutrino Spectra Produced by p-p Interaction in 
Astronomical Environments. ApJ 647,692-708. doi:10.1086/505189, arXiv: astro-ph/0605581.

[49] Karino, S., Miller, J.C., 2016. Accretion mode of the ultraluminous X-ray source M82 X-2. MNRAS 462, 3476-3482. doi:10.1093/mnras/stw1180, arXiv: 1605.05723.

[50] Kawashima, T., Mineshige, S., Ohsuga, K., Ogawa, T., 2016. A radiationhydrodynamics model of accretion columns for ultra-luminous X-ray pulsars. PASJ 68, 83. doi:10.1093/pasj/psw075, arXiv: 1608.04211.

[51] Kawashima, T., Ohsuga, K., Mineshige, S., Yoshida, T., Heinzeller, D., Matsumoto, R., 2012. Comptonized Photon Spectra of Supercritical Black Hole Accretion Flows with Application to Ultraluminous X-Ray Sources. ApJ 752, 18. doi:10.1088/0004-637X/752/1/18.

[52] Kelner, S.R., Aharonian, F.A., Bugayov, V.V., 2006. Energy spectra of gamma rays, electrons, and neutrinos produced at proton-proton interactions in the very high energy regime. Phys. Rev. D 74, 034018. doi:10.1103/PhysRevD.74.034018, arXiv:astro-ph/0606058.

[53] King, A., Lasota, J.P., 2016. ULXs: Neutron stars versus black holes. MNRAS 458, L10-L13. doi:10.1093/mnrasl/slw011, arXiv: 1601.03738.

[54] Kluźniak, W., Lasota, J.P., 2015. An ultraluminous nascent millisecond pulsar. MNRAS 448, L43-L47. doi:10.1093/mnrasl/slu200, arXiv: 1411.1005

[55] Lee, S.H., Ellison, D.C., Nagataki, S., 2012. A Generalized Model of Nonlinear Diffusive Shock Acceleration Coupled to an Evolving Supernova Remnant. ApJ 750, 156. doi:10.1088/0004-637X/750/2/156, arXiv: 1203.3614

[56] Lee, S.H., Patnaude, D.J., Raymond, J.C., Nagataki, S., Slane, P.O., Ellison, D.C., 2015. Modeling Bright $\gamma$-Ray and Radio Emission at Fast Cloud Shocks. ApJ 806, 71. doi:10.1088/0004-637X/806/1/71, arXiv: 1504.05313.

[57] Lockman, F.J., Blundell, K.M., Goss, W.M., 2007. The distance to SS433/W50 and its interaction with the interstellar medium. MNRAS 381, 881-893. doi:10.1111/j.1365-2966.2007.12170.x, arXiv:0707.0506

[58] Lozinskaya, T.A., Moiseev, A.V., 2007. A synchrotron superbubble in the IC10 galaxy: a hypernova remnant? MNRAS 381, L26-L29. doi:10.1111/j.1745-3933.2007.00359.x, arXiv:0708.0626.

[59] Makishima, K., Kubota, A., Mizuno, T., et al., 2000. The Nature of Ultraluminous Compact X-Ray Sources in Nearby Spiral Galaxies. ApJ 535, 632-643. doi:10.1086/308868, arXiv: astro-ph/0001009.

[60] Malkov, M.A., Diamond, P.H., Sagdeev, R.Z., 2011. Mechanism for spectral break in cosmic ray proton spectrum of supernova remnant W44. Nature Communications 2, 194. doi:10.1038/ncomms1195, arXiv: 1004.4714

[61] Mapelli, M., Ripamonti, E., Zampieri, L., Colpi, M., Bressan, A., 2010. Ultra-luminous X-ray sources and remnants of massive metal-poor stars. MNRAS 408, 234-253. doi:10.1111/j.1365-2966.2010.17048.x, arXiv: 1005.3548

[62] Mineo, S., Gilfanov, M., Sunyaev, R., 2012. X-ray emission from star-forming galaxies - I. High-mass X-ray binaries. MNRAS 419, 2095-2115. doi:10.1111/j.1365-2966.2011.19862.x, arXiv: 1105.4610

[63] Mirabel, I.F., Rodríguez, L.F., $1999 . \quad$ Sources of Relativistic Jets in the Galaxy. ARA\&A 37 409-443. doi:10.1146/annurev.astro.37.1.409, arXiv:astro-ph/9902062.

[64] Mizuno, T., Miyawaki, R., Ebisawa, K., et al., 2007. Suzaku Observation of Two Ultraluminous X-Ray Sources in NGC 1313. PASJ 59, 257-267. doi:10.1093/pasj/59.sp1.S257

65] Mori, M., 2009. Nuclear enhancement factor in calculation of Galactic diffuse gamma-rays: A new estimate with DPMJET-3. Astroparticle Physics 31,341-343. doi:10.1016/j . astropartphys.2009.03.004, arXiv:0903.3260

[66] Mushtukov, A.A., Suleimanov, V.F., Tsygankov, S.S., Poutanen, J., 2015. On the maximum accretion luminosity of magnetized neutron stars: connecting X-ray pulsars and ultraluminous X-ray sources. MNRAS 454, 2539-2548. doi:10.1093/mnras/stv2087, arXiv: 1506.03600.

[67] Niino, Y., 2011. Revisiting the metallicity of long-duration gamma-ray burst host galaxies: the role of chemical inhomogeneity within galaxies. MNRAS 417,567-572. doi:10.1111/j.1365-2966.2011.19299.x, arXiv: 1103.1293
[68] Ohira, Y., Murase, K., Yamazaki, R., 2010. Escape-limited model of cosmic-ray acceleration revisited. A\&A 513, A17. doi:10.1051/0004-6361/200913495, arXiv:0910.3449.

[69] Pakull, M.W., Mirioni, L., 2003. Bubble Nebulae around Ultraluminous X-Ray Sources, in: Arthur, J., Henney, W.J. (Eds.), Revista Mexicana de Astronomia y Astrofisica Conference Series, pp. 197-199.

[70] Pakull, M.W., Soria, R., Motch, C., 2010. A 300-parsec-long jet-inflated bubble around a powerful microquasar in the galaxy NGC 7793. Nature 466, 209-212. doi:10.1038/nature09168.

[71] Planck Collaboration, Ade, P.A.R., Aghanim, N., et al., 2016. Planck 2015 results. XIII. Cosmological parameters. A\&A 594, A13. doi:10.1051/0004-6361/201525830, arXiv: 1502.01589.

[72] Poutanen, J., Fabrika, S., Valeev, A.F., Sholukhova, O., Greiner, J., 2013. On the association of the ultraluminous X-ray sources in the Antennae galaxies with young stellar clusters. MNRAS 432, 506-519. doi:10.1093/mnras/stt487, arXiv:1210.1210.

[73] Rolleston, W.R.J., Smartt, S.J., Dufton, P.L., Ryans, R.S.I., 2000. The Galactic metallicity gradient. A\&A 363, 537-554.

[74] Shull, J.M., McKee, C.F., 1979. Theoretical models of interstellar shocks. I - Radiative transfer and UV precursors. ApJ 227, 131-149. doi:10.1086/156712.

[75] Skilling, J., 1975. Cosmic ray streaming. I - Effect of Alfven waves on particles. MNRAS 172, 557-566. doi:10.1093/mnras/172.3.557.

[76] Sol, H., Zech, A., Boisson, C., et al., 2013. Active Galactic Nuclei under the scrutiny of CTA. Astroparticle Physics 43, 215-240. doi:10.1016/j.astropartphys.2012.12.005, arXiv: 1304.3024.

[77] Soria, R., Pakull, M.W., Broderick, J.W., Corbel, S., Motch, C., 2010. Radio lobes and X-ray hotspots in the microquasar S26. MNRAS 409, 541-551. doi:10.1111/j.1365-2966.2010.17360.x, arXiv: 1008.0394

[78] Stecker, F.W., 1970. The Cosmic $\gamma$-Ray Spectrum from Secondary Particle Production in Cosmic-Ray Interactions. Ap\&SS 6, 377-389. doi:10.1007/BF00653856

[79] Stecker, F.W., de Jager, O.C., Salamon, M.H., 1992. TeV gamma rays from 3C 279 - A possible probe of origin and intergalactic infrared radiation fields. ApJL 390, L49-L52. doi:10.1086/186369.

[80] Swartz, D.A., Soria, R., Tennant, A.F., Yukita, M., 2011. A Complete Sample of Ultraluminous X-ray Source Host Galaxies. ApJ 741, 49. doi:10.1088/0004-637X/741/1/49, arXiv:1108.1372.

[81] Swartz, D.A., Tennant, A.F., Soria, R., 2009. Ultraluminous X-Ray Source Correlations with Star-Forming Regions. ApJ 703, 159-168. doi:10.1088/0004-637X/703/1/159, arXiv:0907.4718.

[82] Tsygankov, S.S., Mushtukov, A.A., Suleimanov, V.F., Poutanen, J., 2016. Propeller effect in action in the ultraluminous accreting magnetar M82 X-2. MNRAS 457, 1101-1106. doi:10.1093/mnras/stw046, arXiv: 1507.08288

[83] Uchiyama, Y., Aharonian, F.A., Tanaka, T., Takahashi, T., Maeda, Y., 2007. Extremely fast acceleration of cosmic rays in a supernova remnant. Nature 449, 576-578. doi:10.1038/nature06210.

[84] Wakely, S.P., Horan, D., 2008. TeVCat: An online catalog for Very High Energy Gamma-Ray Astronomy. International Cosmic Ray Conference 3, 1341-1344.

[85] Walton, D.J., Roberts, T.P., Mateos, S., Heard, V., 2011. 2XMM ultraluminous $\mathrm{X}$-ray source candidates in nearby galaxies. $\mathrm{MN}$ RAS 416, 1844-1861. doi:10.1111/j.1365-2966.2011.19154.x, arXiv: 1106.0197

[86] Weaver, R., McCray, R., Castor, J., Shapiro, P., Moore, R., 1977. Interstellar bubbles. II - Structure and evolution. ApJ 218, 377-395. doi:10.1086/155692. 